\title{
Erratum to: Route and survey processing of topographical memory during navigation
}

Luca Latini-Corazzini ${ }^{1}$ - Marie Pascale Nesa ${ }^{2} \cdot$ Mathieu Ceccaldi $^{2,3}$.

Eric Guedj $^{4}$ - Catherine Thinus-Blanc ${ }^{5}$ Franco Cauda ${ }^{1,6} \cdot$ Federico D'Agata $^{1,6,7}$.

Patrick Péruch ${ }^{2}$

Published online: 18 February 2016

(C) Springer-Verlag Berlin Heidelberg 2016

Erratum to: Psychological Research (2010)

\section{4:545-559}

DOI 10.1007/s00426-010-0276-5

Unfortunately, in the original publication, the name of the seventh author was incorrectly published as Federico Dagata. However, the correct name should read as Federico D'Agata.

The online version of the original article can be found under doi:10.1007/s00426-010-0276-5.

Patrick Péruch

patrick.peruch@univmed.fr

1 Dipartimento di Psicologia, Università degli Studi di Torino, Turin, Italy

2 INSERM, U751 Epilepsie and Cognition, Faculté de Médecine de la Timone, Université de la Méditerranée, 27 Bd Jean Moulin, 13385 Marseille Cedex 05, France

3 APHM, Hôpital de la Timone, Service de Neurologie et de Neuropsychologie, Marseille, France

4 APHM, Hôpital de la Timone, Service Central de Biophysique et de Médecine Nucléaire, Marseille, France

5 CNRS, UMR 6146, Université de Provence, Marseille, France

6 CCS fMRI, Ospedale Koelliker, Turin, Italy

7 Dipartimento di Neuroscienze, AOU San Giovanni Battista, Turin, Italy 